\title{
Analytical Theory of Motion of a Mars Orbiter
}

\author{
J.F. San Juan ${ }^{1}$, S. Serrano ${ }^{2}$, and A. Abad ${ }^{2}$ \\ 1 Universidad de La Rioja, 26004 Logroño. \\ 2 Universidad de Zaragoza, 50009 Zaragoza, Spain.
}

\begin{abstract}
The design of spatial missions to Mars requires the development of analytical theories in order to put artificial satellites in orbit around Mars.

In this paper, we present a complete third order analytical model of a satellite perturbed by the zonal $J_{2}, \ldots, J_{6}$ harmonics of the Mars potential. Two Lie transformations, the elimination of the Parallax and the elimination of the Perigee, and the Krylov-Bogoliubov-Mitropolsky method are applied to obtain a complete integration of the model. The algebraic expressions of the generators, the Hamiltonians and the integrals, together with a software code to compute the ephemeris of the satellite, are automatically obtained using our computer algebra system ATESAT.
\end{abstract}

\section{Introduction}

Mars is one of the main challenges for the aerospace community. Since 1965, when the Mariner IV made the first successful flyby of Mars, man has wanted to reach the planet. The study of the Martian topography, gravity surface composition, atmospheric structure, etc., depends on the orbital missions around Mars. Besides, the possibility of future manned missions to Mars forces to select brief parking orbits with a precise analytical determination of the position of the satellite [9].

The interest of the Centre National D'Etudes Spatiales (CNES, France) in this kind of orbiters, together with the experience of the Space Mechanics Group (GME) of the University of Zaragoza in obtaining analytical theories of Earth artificial satellites by using symbolic computational tools, drove us to collaborate in order to obtain an analytical theory of a Martian artificial satellite with an error less than one kilometer in one month. To this aim, we used the same methods as those applied to Earth satellites.

The Lie-Deprit method [7] based on Lie canonical transformations was used to develop efficient analytical theories of the satellite problem. To obtain our theory we applied two Lie transformations: the elimination of the Parallax and the elimination of the Perigee. After these eliminations, the simplified Hamiltonian was reduced to one degree of freedom. To obtain a theory without singularities in eccentricity and inclination and without series expansions in eccentricity, we applied the Krylov-Bogoliubov-Mitropolsky (KBM) method instead of the classical Delaunay normalization. Both, the Lie-Deprit method and the KBM technique are very well suited methods for symbolic computation. 
In general, during the computing of an analytical theory in the artificial satellite problem one has to cope with long series expansion, handling a huge amount of terms and particular objects like the so-called Poisson serie\&1. This makes general purpose algebraic manipulators ineffective and forces to create specific computer algebra systems to handle these type of objects in an efficient way. Our software ATESAT ([2313]) uses the Poisson Series Processor PSPC [114] to generate automatically analytical theories and their corresponding ephemeris programs for satellite orbits.

With ATESAT we developed the complete third order theory of the motion of an artificial satellite perturbed by the first sixth order zonal harmonics of the potential of Mars. With this theory we obtained the required precision; in fact the error for a wide range of initial conditions is less than 400 meters per month.

\section{Dynamical Model}

The Hamiltonian formalism that describes the orbit of an artificial satellite around Mars is written in Whittaker's or polar-nodal variabled as

$$
\mathcal{H}=\sum_{n \geq 0} \frac{\epsilon^{n}}{n !} \mathcal{H}_{n}
$$

with

$$
\begin{array}{rlrl}
\mathcal{H}_{0} & =\frac{1}{2}\left(R^{2}+\frac{\Theta^{2}}{r^{2}}\right)-\frac{\mu}{r}, & \mathcal{H}_{1}=\frac{\mu}{r}\left(\frac{\alpha}{r}\right)^{2} P_{2}\left(s_{i} \sin \theta\right), \\
\mathcal{H}_{2}=2 \frac{\mu}{r} \sum_{n \geq 3}^{6}\left(\frac{\alpha}{r}\right)^{n} \frac{J_{n}}{J_{2}^{2}} P_{n}\left(s_{i} \sin \theta\right), & \mathcal{H}_{k}=0, \quad k \geq 3,
\end{array}
$$

where $P_{n}$ is the Legendre polynomial of degree $n, \mu$ is the gravitational constant, $\alpha$ is the equatorial radius, $J_{n}$ are the zonal harmonic coefficients, $s_{i}=\sin i=$ $\sqrt{1-\left(N^{2} / \Theta^{2}\right)}$, is a function of the momenta $N$ and $\Theta$, and the small parameter $\epsilon$ is the Martian constant of oblateness.

The variable $\nu$ is cyclic in the zonal problem, and therefore we have a dynamical system with two degrees of freedom.

${ }^{1}$ A Poisson series is a multivariate Fourier series, whose coefficients are multivariate Laurent series

$$
\sum_{i_{0}, \ldots, i_{n-1}, j_{0}, \ldots, j_{m-1}} C_{i_{0}, \ldots, i_{n-1}}^{j_{0}, \ldots, j_{m-1}} x_{0}^{i_{0}} \ldots x_{n-1}^{i_{n-1}}\left(\begin{array}{c}
\sin \\
\cos
\end{array}\right)\left(j_{0} y_{0}+\ldots+j_{m-1} y_{m-1}\right) .
$$

$2(r, \theta, \nu, R, \Theta, N)$, where $r$ is the radial distance from the planet's mass center to the satellite, $\theta$ is the argument of latitude, and $\nu$ is the argument of the ascending node. The variables $R, \Theta$ and $N$ are the conjugate momenta to the coordinates $r, \theta$ and $\nu$, respectively. 


\section{Canonical Lie Transformations}

A Lie transformation 7,1011 can be defined as an infinitesimal contact transformation $\varphi:(\boldsymbol{y}, \boldsymbol{Y}, \epsilon) \rightarrow(\boldsymbol{x}, \boldsymbol{X})$, such that $\boldsymbol{x}(\boldsymbol{y}, \boldsymbol{Y} ; \epsilon), \boldsymbol{X}(\boldsymbol{y}, \boldsymbol{Y} ; \epsilon)$ satisfy the differential equations

$$
\frac{d \boldsymbol{x}}{d \epsilon}=\nabla_{\boldsymbol{X}} W(\boldsymbol{x}, \boldsymbol{X}, \epsilon), \quad \frac{d \boldsymbol{X}}{d \epsilon}=-\nabla_{\boldsymbol{x}} W(\boldsymbol{x}, \boldsymbol{X}, \epsilon),
$$

with the initial conditions $\boldsymbol{x}(\boldsymbol{y}, \boldsymbol{Y}, \epsilon=0)=\boldsymbol{y}, \boldsymbol{X}(\boldsymbol{y}, \boldsymbol{Y}, \epsilon=0)=\boldsymbol{Y}$, where $\boldsymbol{x}, \boldsymbol{X}, \boldsymbol{y}, \boldsymbol{Y} \in \mathbb{R}^{m}$, and $W=\sum_{i \geq 0}\left(\epsilon^{i} / i !\right) W_{i+1}(\boldsymbol{x}, \boldsymbol{X})$ is the generator of the transformation.

In the particular case of the Hamiltonian formalism, a Lie transformation converts a Hamiltonian $\mathcal{H}(\boldsymbol{x}, \boldsymbol{X} ; \epsilon)=\sum_{i>0}\left(\epsilon^{i} / i !\right) \mathcal{H}_{i, 0}(\boldsymbol{x}, \boldsymbol{X})$ into a new one $\mathcal{K}(\boldsymbol{y}, \boldsymbol{Y} ; \epsilon)=\sum_{i \geq 0}\left(\epsilon^{i} / i !\right) \mathcal{H}_{0, i}(\boldsymbol{y}, \boldsymbol{Y})$ by means of the relations

$$
\mathcal{H}_{p, q}=\mathcal{H}_{p+1, q-1}+\sum_{k=0}^{p}\left(\begin{array}{l}
p \\
k
\end{array}\right)\left(\mathcal{H}_{p-k, q-1} ; W_{k+1}\right),
$$

where $\left({ }_{-} ;-\right)$stands for the Poisson bracket. Equation (4) is called Lie triangle. Note that with this approach, there appear many intermediate terms $\mathcal{H}_{p, q}$ which must be computed and stored.

The Lie-Deprit method [7] tries to find the generator of a Lie transformation to turn the Hamiltonian into a new one satisfying some prefixed conditions.

This method looks for the generator order by order. Once the order $(n-1)$ is solved we know the expressions of $W_{i}, i=0, \ldots, n-1$ and $\mathcal{H}_{p, q}, p+q \leq n-1$. By joining the equations in (4) for $p+q=n$, we find the homological equation

$$
\mathcal{L}_{0} W_{n}=\left(\mathcal{H}_{0}, W_{n}\right)=\mathcal{H}_{0, n}-\tilde{\mathcal{H}}_{n, 0},
$$

where $\tilde{\mathcal{H}}_{n, 0}$ can be computed from the previous orders by means of the Lie triangle. To solve the order $n$, we must follow three steps:

1. Compute $\tilde{\mathcal{H}}_{n, 0}$ from the expressions of order $(n-1)$.

2. Choose $\mathcal{H}_{0, n}$.

3. Find an integral $W_{n}$ of the homological equation.

\subsection{Elimination of the Parallax}

The first step of this theory looks for a Lie transformation called elimination of the Parallax. This canonical transformation [8] reduces the complexity, not the number of degrees of freedom, of the Hamiltonian (11). Besides, the elimination of the Parallax algorithm allows us to compute the expression in a close form of the eccentricity, and therefore to obtain general purpose theories valid for any kind of elliptic eccentricity.

The homological equation (5) is expressed, in Whittaker variables, as follow

$$
\mathcal{L}_{0} \mathcal{W}_{n}=R \frac{\partial \mathcal{W}_{n}}{\partial r}-\left(\frac{\mu}{r^{2}}-\frac{\Theta^{2}}{r^{3}}\right) \frac{\partial \mathcal{W}_{n}}{\partial R}+\frac{\Theta}{r^{2}} \frac{\partial \mathcal{W}_{n}}{\partial \theta}=\mathcal{H}_{0, n}-\tilde{\mathcal{H}}_{n, 0} .
$$


Instead of looking for an integral of the previous equation, we will apply the property

$$
\mathcal{L}_{0}\left(\sum_{j \geq 0}\left(C_{j} \sin j \theta+S_{j} \cos j \theta\right)\right)=\frac{\Theta}{r^{2}} \sum_{j \geq 1} j\left(C_{j} \cos j \theta-S_{j} \sin j \theta\right),
$$

valid for any function of the algebra $\mathcal{F}=\left\{F=\sum_{j \geq 0}\left(C_{j} \cos j \theta+S_{j} \sin j \theta\right)\right.$, $\left.C_{j}, S_{j} \in \operatorname{ker}\left(\mathcal{L}_{0}\right)\right\}$. Particularly, using the $C=e \cos g, S=e \sin g$ and $p=$ $a\left(1-e^{2}\right)$ functions of the orbital elements $a, e$ and $g$, the Hamiltonian (1) can be expressed as a function of $\mathcal{F}$ if we take into account the relations $1 / r=1 / p+$ $C / p \cos \theta+S / p \sin \theta, R=C \Theta / p \sin \theta-S \Theta / p \cos \theta$, since $C, S, p, \Theta \in \operatorname{ker}\left(\mathcal{L}_{0}\right)$.

After applying the previous change of variables, the equation (6) becomes

$$
\mathcal{L}_{0}\left(\mathcal{W}_{n}\right)+\frac{\Theta}{r^{2}} C_{0}=\frac{\Theta}{r^{2}} F
$$

and then we apply the three steps of the method described in the previous section

1. Computing $F=\left(r^{2} / \Theta\right) \tilde{\mathcal{H}}_{n, 0}=\sum_{j \geq 0}\left(C_{j} \cos j \theta+S_{j} \sin j \theta\right)$.

2. Choosing $\mathcal{H}_{0, n}=\left(\Theta / r^{2}\right) C_{0}$.

3. Finding $\quad \mathcal{W}_{n}=\sum_{j \geq 1}\left[\left(C_{j} / j\right) \sin j \theta-\left(S_{j} / j\right) \cos j \theta\right]$.

Note that after computing $\tilde{\mathcal{H}}_{n, 0}$ by means of the Lie triangle, obtaining $\mathcal{H}_{0, n}$ and $\mathcal{W}_{n}$ becomes a simple symbolic exercise of coefficients reordering.

After the Parallax elimination, the transformed Hamiltonian and the generator of third order, expressed as a Poisson Series, have 176 and 1837 terms, respectively. The number of terms of the direct and inverse expression of this transformation are given in the following table

\begin{tabular}{ccccccc}
\hline Parallax & $r$ & $\theta$ & $\nu$ & $R$ & $\Theta$ & $N$ \\
\hline Direct & 2056 & 5000 & 4738 & 1988 & 3057 & 1 \\
Inverse & 2052 & 4981 & 4610 & 1989 & 3034 & 1
\end{tabular}

\subsection{Elimination of the Perigee}

Apparently, the elimination of the Parallax removes the argument of latitude $\theta$, however, this variable appears implicitly in the state functions $C$ and $S$, which are functions of the perigee $g$. In order to reduce the problem, we can eliminate the perigee by applying a new Lie transformation whose generator has two terms

$$
\mathcal{W}_{n}=\mathcal{W}_{n}^{*}(C, S, \Theta, \theta)+\tilde{\mathcal{W}}_{n}\left(C, S, \Theta,_{-}\right),
$$

$\mathcal{W}_{n}^{*}$, which depends on $\theta$, and $\tilde{\mathcal{W}}_{n}$, which does not depend on $\theta$. The Lie operator $\mathcal{L}$ applied to this generator

$$
\mathcal{L}_{0} \mathcal{W}_{n}=R \frac{\partial \mathcal{W}_{n}}{\partial r}-\left(\frac{\mu}{r^{2}}-\frac{\Theta}{r^{3}}\right) \frac{\partial \mathcal{W}_{n}}{\partial R}+\frac{\Theta}{r^{2}} \frac{\partial \mathcal{W}_{n}}{\partial \theta}=\frac{\Theta}{r^{2}} \frac{\partial \mathcal{W}_{n}^{*}}{\partial \theta},
$$

only depends on $\mathcal{W}_{n}^{*}$. 
With such a property, the solution $\mathcal{W}_{n}^{*}=\int r^{2}\left(\tilde{\mathcal{H}}_{n, 0}-\mathcal{H}_{0, n}\right) / \Theta d \theta$ of the homological equation only gives one part of the expression of the generator.

The homological equation (5) is obtained by detaching from the $n$-th line of the Lie triangle the known terms of order $n-1$ of the unknown terms. Then, there are more unknown terms depending on $\tilde{\mathcal{W}}_{n-1}$, and the homological equation becomes $\mathcal{L}_{0} \mathcal{W}_{n}^{*}=\tilde{\mathcal{H}}_{n, 0}+\mathcal{H}_{0, n}+n\left(\mathcal{H}_{1,0} ; \tilde{\mathcal{W}}_{n-1}\right)$.

By computing $\tilde{\mathcal{H}}_{n, 0}$ and splitting it into the part $\tilde{\mathcal{H}}_{n, 0}^{\theta}$ that depends on $\theta$ and the part $\tilde{\mathcal{H}}_{n, 0}^{*}$ that does not depends on $\theta$, we can choose the new Hamiltonian of order $n>1$ as the expression

$$
\mathcal{H}_{0, n}=\left\langle\tilde{\mathcal{H}}_{n, 0}^{*}\right\rangle_{g}=\frac{1}{2 \pi} \int_{0}^{2 \pi} \tilde{\mathcal{H}}_{n, 0}^{*}(C, S) d g,
$$

that does not depends on $g$.

Eventually, taking into account the expression

$$
\left(\mathcal{H}_{1,0} ; \tilde{\mathcal{W}}_{n-1}\right)=\mathcal{F}_{1}(\theta)-\frac{3 \mu \alpha^{2}}{2 \Theta^{3} r^{2}}\left(4-5 s_{i}^{2}\right) \frac{\partial \tilde{\mathcal{W}}_{n-1}}{\partial g}
$$

we can use it to obtain $\tilde{\mathcal{W}}_{n-1}$

$$
\tilde{\mathcal{W}}_{n-1}=\frac{2 \Theta^{3} r^{2}}{3 n \mu \alpha^{2}} \frac{1}{\left(4-5 s_{i}^{2}\right)} \int\left(\mathcal{H}_{0, n}-\left\langle\tilde{\mathcal{H}}_{n, 0}^{*}\right\rangle_{g}\right) d g
$$

and $\mathcal{W}_{n}^{*}$

$$
\mathcal{W}_{n}^{*}=\int \frac{r^{2}}{\Theta}\left(\tilde{\mathcal{H}}_{n, 0}^{\theta}+n \mathcal{F}_{1}(\theta)\right) d \theta .
$$

This algorithm called elimination of the Perigee [5] is applied to remove the argument of the perigee $g$ from the perturbation. This elimination is not a normalization in the sense that the transformed Hamiltonian does not belong to the kernel of the Lie derivative $\mathcal{L}_{0}$ associated with $\mathcal{H}_{0}$, rather it reduces by one the number of degrees of freedom.

The Hamiltonian after the two previous transformations can be expressed as

$$
\mathcal{H}=\frac{1}{2}\left(R^{2}+\frac{\Theta^{2}}{r^{2}}\right)-\frac{\mu}{r}+\sum_{n \geq 1} \frac{\epsilon^{n}}{n !} \mathcal{M}_{n}^{i, j} \frac{R^{i}}{r^{j}},
$$

where $\mathcal{M}_{n}^{i, j}$ are functions of the constants $\alpha$ and $\mu$ and the momenta $\Theta$ and $N$. This transformed Hamiltonian has 317 terms, and the generator has 4521 terms. The number of terms of the direct and inverse expression of this transformation, which is considerably greater than in the previous transformation, is given in the following table

\begin{tabular}{ccccccc}
\hline Perigee & $r$ & $\theta$ & $\nu$ & $R$ & $\Theta$ & $N$ \\
\hline Direct & 83410 & 155376 & 81893 & 80156 & 66322 & 1 \\
Inverse & 83294 & 154769 & 81435 & 79915 & 66322 & 1
\end{tabular}




\section{The Satellite as a Perturbed Harmonic Oscillator}

The variables $\theta$ and $\nu$ are cyclic in the Hamiltonian (7), then the momenta $\Theta$ and $N$ are constant and we will consider only the two first Hamilton's equations

$$
\frac{d r}{d t}=\frac{\partial \mathcal{H}}{\partial R}, \quad \frac{d R}{d t}=-\frac{\partial \mathcal{H}}{\partial r},
$$

in order to find the temporal evolution of $r$ and $R$. After finding the solution of previous differential equations, the problem lies in the computation of two quadratures to obtain $\theta$ and $\nu$.

By differentiating again the first equation in (8), combining both to obtain a second order differential equation, and changing the variables $r$ and $d r / d t$ and the time $t$ for two new variables $u$ and $v$, and a new time $s$ defined by

$$
u=\frac{1}{r}-\frac{\mu}{\Theta^{2}}, \quad r^{2} \frac{d s}{d t}=\Theta, \quad v=\frac{d u}{d s},
$$

we obtain the equation of a perturbed harmonic oscillator

$$
\frac{d^{2} u}{d s^{2}}+u=\sum_{n \geq 1} \frac{\epsilon^{n}}{n !} \mathcal{K}_{n}^{i, j} u^{i} v^{j}
$$

where $\mathcal{K}_{n}^{i, j}$ are polynomials in the constants $\mu, \Theta$ and $\mathcal{M}_{m}^{i, j}$.

\subsection{The Krylov-Bogoliubov-Mitropolski (KBM) Method}

The solution of the differential equation (10) of the perturbed harmonic oscillator can be written as an asymptotic expansion $u=\sum_{n \geq 0}\left(\epsilon^{n} / n !\right) u_{n}(\delta, f)$, where $u_{0}=\delta \cos f, u_{n}(\delta, f)$ are $2 \pi$-periodic functions in $f$, and the variation of $\delta$ and $f$ with respect to the time is given by

$$
\frac{d \delta}{d s}=\sum_{n \geq 0} \frac{\epsilon^{n}}{n !} A_{n}(\delta), \quad \frac{d f}{d s}=\sum_{n \geq 0} \frac{\epsilon^{n}}{n !} B_{n}(\delta), \quad A_{0}=0, \quad B_{0}=\omega .
$$

The KBM method, 612], gives an iterative way to find the terms $A_{n}, B_{n}$ and $u_{n}$. Assuming that the order $(n-1)$ has been solved, we find a function $U_{n}$ that depends on all expressions until order $(n-1)$. Then, by calling $c_{j}(F)$ and $s_{j}(F)$ respectively the coefficients of $\cos j f$ and $\sin j f$ in the Fourier expansion of $F(f)$, the expressions of $A_{n}, B_{n}$ are $A_{n}=-s_{1}\left(U_{n}\right) /(2 \omega), B_{n}=-c_{1}\left(U_{n}\right) /(2 \omega \delta)$, and the function $u_{n}$ is given by its Fourier expansion whose coefficients are $c_{0}\left(u_{n}\right)=c_{0}\left(U_{n}\right) / \omega^{2}, c_{j}\left(u_{n}\right)=c_{j}\left(U_{n}\right) / \omega_{j}, s_{j}\left(u_{n}\right)=s_{j}\left(U_{n}\right) / \omega_{j}, j \geq 2$, with $\omega_{j}=\omega^{2}\left(1-j^{2}\right)$.

Applying the KBM method to the equation (10) we find the variation of $\delta$ and $f$ with respect to the new time $s$ by means of the expressions

$$
\frac{d \delta}{d s}=0, \quad \frac{d f}{d s}=n_{f}=1+\sum_{n \geq 1} \frac{\epsilon^{n}}{n !} \Psi\left(\delta, \mathcal{K}_{n}^{i, j}\right) .
$$


Equation (12) shows that $\delta$ has a constant value. $n_{f}$ is also a constant since the values of $\mathcal{K}_{n}^{i, j}$ are constant. However, we will not integrate equation (12) to obtain the relation between $f$ and $s$, rather, we will obtain a generalized Kepler equation in the next section. This latter equation will give us a direct relation between $f$ and $t$.

Besides we obtain the expressions of $u$ and $v$. These expressions together with the change of variables (9), allow us to find the expression of $R$

$$
R=\frac{\mu e}{\Theta} \sin f-\epsilon \mathcal{K}_{1}^{1,0} \frac{\mu e}{\Theta} \sin f+\frac{\epsilon^{2}}{2 !} \sum_{k=1}^{5} \mathcal{K}_{k}^{R} \sin k f,
$$

and the expression of $1 / r$, that once inverted gives

$$
\begin{aligned}
r= & \frac{p}{1+e \cos f}-\epsilon \frac{p^{2} \mathcal{K}_{1}^{0,0}}{(1+e \cos f)^{2}}+\frac{\epsilon^{2}}{2 !}\left[\frac{2 p^{3}\left(\mathcal{K}_{1}^{0,0}\right)^{2}}{(1+e \cos f)^{3}}\right. \\
& \left.-\frac{p^{2}}{(1+e \cos f)^{2}}\left(\mathcal{K}_{0}^{r}+\mathcal{K}_{2}^{r} \cos 2 f+\mathcal{K}_{3}^{r} \cos 3 f+\mathcal{K}_{4}^{r} \cos 4 f+\mathcal{K}_{5}^{r} \cos 5 f\right)\right],
\end{aligned}
$$

where $\mathcal{K}_{k}^{R}$ and $\mathcal{K}_{k}^{r}$ are functions of constants, and we use the generalized semilatus rectum, eccentricity and semi-mayor axis. These expressions are used to obtain the variables $\theta$ and $\nu$ and the momenta $\Theta$ and $N$.

\section{Generalized Kepler Equation}

The variation of the generalized true anomaly, $f$, with respect to time, $t$, is obtained after considering the definition of $s$ given by (9) from which we get

$$
n_{f} \Theta d t=r^{2} d f
$$

in which we substitute $r$ by the expression (14) in terms of $f$.

Then, we apply a change of variables as in the case of the transformation of the true anomaly into the eccentric anomaly in the non-perturbed two body problem $p /(1+e \cos f)=a(1-e \cos E), d f=\sqrt{1-e^{2}}(1-e \cos E) d E$. This change of variables is usually used in orbital mechanics to transform integrals with powers of $(1+e \cos f)$ in the denominator into terms with powers of $(1-e \cos E)$ in the numerator. By doing so, we can integrate these expressions. In our problem, the existence of factors $\cos n f, \sin n f$ in the numerator of the perturbation terms implies that, even after applying the change, some terms still have powers of $(1-e \cos E)$ in the denominator.

A practical way to avoid this formal problem is to apply first the change of $f$ into $w$ defined by $w=1+e \cos f, \cos f=(w-1) / e$. By using the properties of Chebyshev's polynomials, $T_{n}, U_{n}$, we can express each term of (15) as a positive or negative power of $w$, multiplied or not by $\sin f$. Then, to integrate (15) we 
just have to consider integrals of the form $\int w^{n} d f, \int w^{n} \sin f d f$, that can be easily performed [4].

Finally, we obtain the equation $n(t-T)=E-e \sin E+\sum_{i>0}\left(\epsilon^{i} / i !\right) \mathcal{K}_{i}(E, f)$, where $T$ represents the value of $t$ when $f=E=0$, equation known as the generalized Kepler's equation. Note that for orders greater than one, some terms in $f$ and $E$ are mixed up in this generalized Kepler equation.

Acknowledgements. We are very grateful to I. Tijera for her suggestions. This work has been supported in part by the Ministerio de Educación y Ciencia (DGICYT BFM2002-03157 and \# BFM2003-02137) and the Department of Space Mathematics of Centre National d'Etudes Spatiales (France).

\section{References}

1. Abad, A. and San Juan, J. F.: PSPC: A Poisson Series Processor coded in C. Dynamics and Astrometry of Natural and Artificial Celestial Bodies. Kurzynska et al eds. Poznam, Poland, (1993), 383-389.

2. Abad, A. and San Juan, J. F.: ATESAT: software tool for obtaining automatically ephemeris from analytical simplifications. Conseil de L'Europe. Cahiers du Centre Européen de Géodynamique et de Séismologie. A. Elipe and P. Paquet eds. Luxembourg. 10, (1995), 93-98.

3. Abad, A., Elipe, A., Palacián, J. and San Juan, J. F.: ATESAT: A Symbolic Processor for Artificial Satellite Theory. Mathematics and Computers in Simulation. 45, (1998), 497-510.

4. Abad, A., San Juan, J. F., and Gavín, A.: Short term evolution of artificial satellites. Celestial Mechanics and Dynamical Systems. 79, (2001), 277-296.

5. Alfriend, K. T. and Coffey, S. L.: Elimination of the Perigee in Satellite Problem, Celestial Mechanics, 32, (1984), 163-172.

6. Bogoliubov, N. N. and Mitropolsky, Y. A.: Asymptotic Method in the Theory of Nonlinear Oscillations, Gordon and Breach. New York, (1961).

7. Deprit, A.: Canonical Transformations Depending on a Small Parameter, Celestial Mechanics, 1, (1969), 12-30.

8. Deprit, A.: The Elimination of the Parallax in Satellite Theory. Celestial Mechanics, 24, (1981), 111-153.

9. Desai, P. N., Braun, R. D. and Powell, R. W.: Aspects of Parking Orbit Selection in a Manned Mars Mission, NASA TP-3256, (1992).

10. Henrard, J.: On a perturbation theory using Lie Transform, Celestial Mechanics, 3, (1970), 107-120.

11. Kamel, A. A.: Perturbation methods in the theory of nonlinear oscillations, Celestial Mechanics, 3, (1970), 90-106.

12. Krylov, N. and Bogoliubov, N. N.: Introduction to Nonlinear Mechanics. Princeton University Press, Princeton N.Y., (1947).

13. San Juan, J. F.: ATESAT: Automatization of theories and ephemeris in the artificial satellite problem, Tech. rep. CT/TI/MS/MN/94-250, CNES, France, (1994).

14. San Juan, J. F.: Manipulación algebraica de series de Poisson. Aplicación a la teoría del satélite artificial. Ph. D. Dissertation, Univ. of Zaragoza, (1996). 